\title{
Color and visual complexity in abstract images: Part II
}

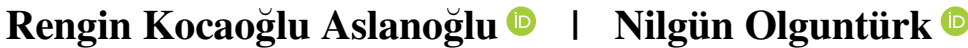

Department of Interior Architecture and Environmental Design, Bilkent University, Ankara, Turkey

\section{Correspondence}

Rengin Kocaoğlu Aslanoğlu, Department of Interior Architecture and Environmental Design, Bilkent University, Ankara, Turkey. Email: rengin.kocaoglu@bilkent.edu.tr

\begin{abstract}
There are a limited number of studies examining color, visual complexity, and visual interest together, and one of the recent studies that tried to bring a new understanding to the association between color, visual complexity, and visual interest was the first part of the current study. Most of the well-known color studies researching the effects of color on psychology, physiology, emotion, mood, attention, well-being, visual complexity, and visual interest used isolated color patches that might be lacking in reflecting the dominating factors. Thus, the aim of this study was to find the relationship between visual complexity, visual interest, and color difference $(\Delta E)$ values of colors in abstract images, and it was hypothesized that, as the average $\Delta E$ value of colors in an abstract image increases, visual interest and visual complexity will increase until reaching a threshold where visual interest and visual complexity start to decrease. In order to test the hypothesis, a new abstract image was generated and colored. The generated abstract image was rated by 120 undergraduate students from the Faculty of Art, Design and Architecture. As the results of the study indicated, there was an inverted U-curve relationship between average $\Delta E$ values and both visual interest and visual complexity in abstract images.
\end{abstract}

\section{K E Y W O R D S}

abstract images, color, visual complexity, visual interest

\section{1 | INTRODUCTION}

Everybody experiences and encounters color both in natural and artificial environments every day. Colors are never seen in isolation because the environment that surrounds us presents colors together with other colors. ${ }^{1}$ Most of the well-known color studies researching the effects of color on psychology, physiology, emotion, mood, attention, and well-being used isolated color patches, which might not reflect the dominating factors. There are a limited number of studies examining color, visual complexity, and visual interest together, and one of the recent studies that attempted to bring a new understanding to the association between color, visual complexity, and visual interest was the first part of this current study. ${ }^{2}$ Most of the results of complexity studies vary since they manipulated all the complexity dimensions without considering the dimensions' appropriateness to the related factors. ${ }^{3}$ The first part of this study framed the dimensions of visual complexity by focusing only on unintelligibility, disorganization, and the variety of colors in abstract images. Abstract images were selected to evaluate the association between color, visual interest, and visual complexity because a visual language is composed in those images by only using line, form, shape, and color, which makes it universal and culture-independent. In the first part of this study, Piet Mondrian's two abstract paintings, Ocean 5 and Composition No. VII, and Vasily Kandinsky's two abstract paintings, Composition 8 and Decisive Rose, were selected. $^{2}$ According to the results of the first part, abstract images that were evaluated as visually complex and interesting were the ones that had less difficulty in differentiating hues (intelligible). As the selected images are complex in 
nature, their degrees of complexity demonstrated that they became unintelligible, that is, when viewers have difficulty in distinguishing hues, they start losing their visual interest. ${ }^{2}$ This particular study, with the guidance of Part I, found the dominating dimension of complexity and delved deeper into the role of color in visual complexity.

In order to gain an in-depth knowledge about the relationship between color, visual complexity, and visual interest, an abstract image was generated and colored according to various color schemes: monochromatic, analogous, splitcomplementary, complementary, triadic, and tetradic. Those color schemes are used by designers and artists and are mostly intuitive in their works and described qualitatively. For the current study, the qualitatively described color schemes were interpreted as $\Delta E$ values, which are the quantitative values of the color difference in the generated abstract image. The aim of this study was to find the relationship between visual complexity, visual interest, and the color difference $(\Delta E)$ in abstract images. It was hypothesized that, as the average $\Delta E$ value of color difference in an abstract image increases, visual interest and visual complexity will increase until reaching a threshold where visual interest and visual complexity start to decrease. In other words, a graphical analysis shows there is an inverted U-curve relationship between average $\Delta E$ values and both visual interest and visual complexity in abstract images.

\section{2 | METHOD}

According to the results of the experiment of Part I, the second experiment was designed to understand the role of color in visual complexity. As the results of the first experiment demonstrate, participants rated Vasily Kandinsky's Composition 8 as the most visually complex, visually interesting, and most intelligible. ${ }^{2}$ Participants also rated Piet Mondrian's
Ocean 5 as the least visually complex, least visually interesting, and the least intelligible. After examining the results of the previous study, a new abstract image (see Figure 1) was generated using Adobe Photoshop CS6. The new generated abstract image provided the opportunity to examine only the effects of color on visual complexity and visual interest by controlling all other parameters.

\section{1 | Analyzing Kandinsky's Composition 8 and preparing the Kandinsky-like abstract image}

According to the results of the first experiment, Composition 8 by Vasily Kandinsky, which was rated as the most visually complex, was selected for this study to create a new similar abstract image. We created a Kandinsky-like abstract image as the aim of this study was to understand the role of color in determining the level of visual complexity in abstract images. The generated abstract image could be called "Abstract Plasticism," similar to Vasily Kandinsky's works, which consisted of geometric elements and straight lines colored with flat colors as Kandinsky's Composition 8 includes 103 different colors with different geometric shapes such as triangles, circles, rectangles, and polygons varying in size, orientation, and allocation. The new generated abstract image was as visually complex and interesting as the original one because it included the same proportions and the same geometric shapes at the same allocations as the original one (See Figure 1). Hence, the generated abstract image can be called the resembled reproduction of Composition 8, that is, a Kandinsky-like abstract image that contained 106 geometric shapes and 106 different colors.

For the new generated abstract image, colors were selected from the International Commission on Illumination (CIE) Chromaticity Chart within the Wide Gamut Red, Blue, Green (RGB) (see Figure 2). The CIE Chromaticity Chart, which has a horse shoe-shaped curve, is a diagram to visualize the color

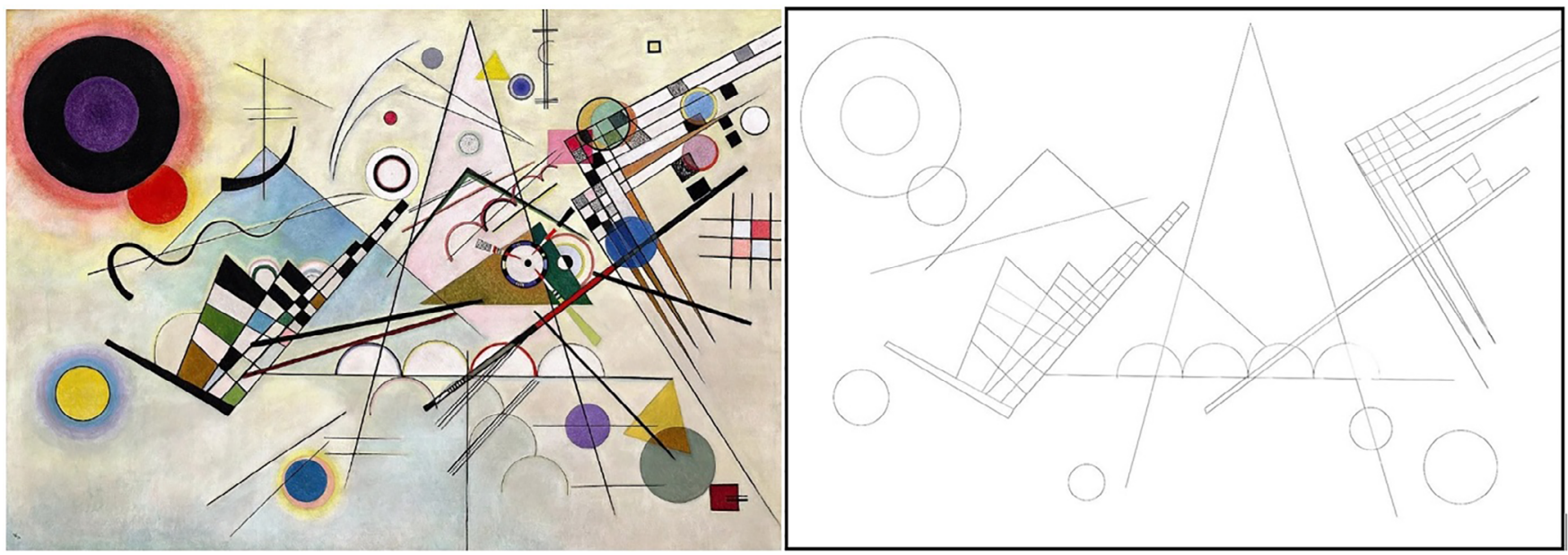

F I G URE 1 The original Composition 8 (left) and the generated abstract image (right) 
F I G URE 2 Left: The CIE

Chromaticity Chart and six zones (white triangle shows Wide Gamut RGB). Right: A close look at one of the origins of RGB data $(R: 254, G: 172, B: 40)$ selected from zone number 6

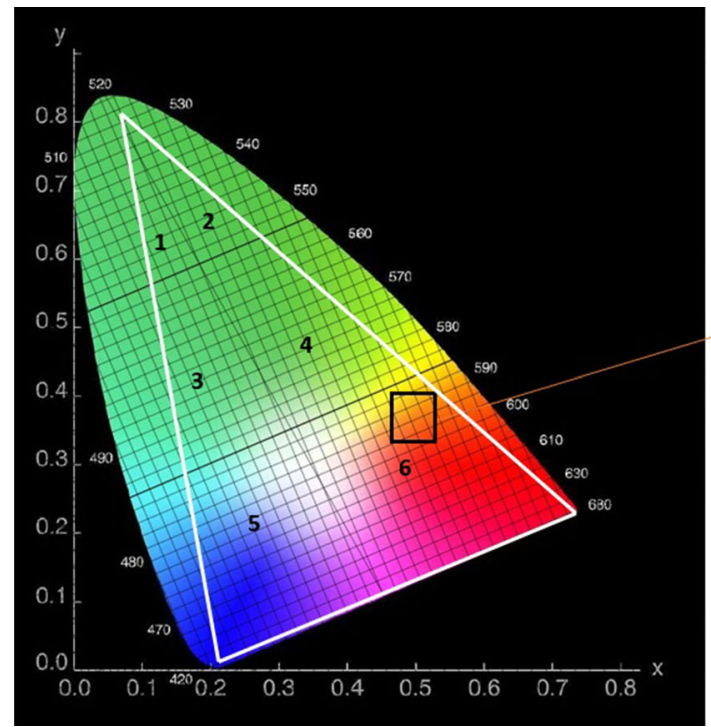

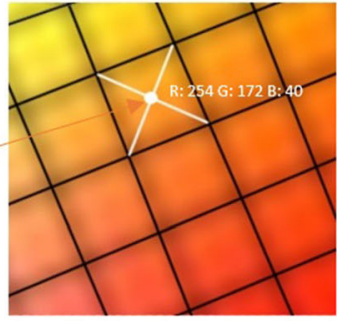

space. This diagram was also used as a visualization model to understand the way color choices were made for this study. In order to have 106 different colors for each generated abstract image, the CIE Chromaticity Chart was divided into six zones to include all the colors presented in the chart. The horse shoeshaped curve was first divided into half and was then divided again with two perpendicular lines, producing six equivalent zones (see Figure 2). With this particular method, each zone has an equivalent number of colors. Different colors can also be obtained by this way of zonal division.

By using all six zones of the CIE Chromaticity Chart, 19 zone combinations were obtained (1-6) for coloring the generated abstract image. As there are 106 colors in the abstract composition, 53 colors were selected from one zone (eg, zone number 2 starting from the square at right bottom corner to left upper corner), and the other 53 colors were selected from another zone (eg, zone number 5 starting from the square at left bottom corner to right upper corner). Each of the 53 colors was selected from the centers of equally divided squares as shown in the Figure 2. The method of selecting colors from the furthest corners maximizes the proximities between colors having varying $\Delta E$ values. In order to understand if there was an effect of the allocation of colors on the abstract image, three abstract images were colored by using the same zones but in reverse order (2-6, 3-5, 4-6, 5-3, 5-6, 6-2, 6-4, and 6-5). The background color of each abstract image was a mid-lightness value $(L: 50, a: 0, b: 0)$ (see Table 2$)$.

\section{2 | Color difference}

$\Delta E$ indicates the perceived total color difference between two colors. In other words, it is the measure of the difference in visual perception of two given colors. $\Delta$ is a mathematical term meaning difference in a variable, and $E$ is derived from a German word Empfindung, which means sensation. ${ }^{4,5} \Delta E$ is a metric for understanding how the human eye perceives color difference, which can be calculated by the CIEDE2000 equation (see Equation 1). ${ }^{4-6}$ This equation considers all color components together. The CIEDE2000 Color Difference Formula is given in Equation (1).

$$
\begin{gathered}
\Delta L^{\prime}=L_{2}^{*}-L_{1}^{*} \\
\bar{L}=\frac{L_{1}^{*}+L_{2}^{*}}{2} \quad \bar{C}=\frac{C_{1}^{*}+C_{2}^{*}}{2}
\end{gathered}
$$

$a_{1}^{\prime}=a_{1}^{*}+\frac{a_{1}^{*}}{2}\left(1-\sqrt{\frac{\bar{C}^{7}}{\bar{C}^{7}+25^{7}}}\right) \quad a_{2}^{\prime}=a_{2}^{*}+\frac{a_{2}^{*}}{2}\left(1-\sqrt{\frac{\bar{C}^{7}}{\bar{C}^{7}+25^{7}}}\right)$

$$
\begin{aligned}
& \bar{C}^{\prime}=\frac{C_{1}^{\prime}+C_{2}^{\prime}}{2} \text { and } \Delta C^{\prime}=C_{2}^{\prime}-C_{1}^{\prime} \text { where } \\
& C_{1}^{\prime}=\sqrt{a_{1}^{\prime 2}+b_{1}^{* 2}}, C_{2}^{\prime}=\sqrt{a_{2}^{\prime 2}+b_{2}^{* 2}} \\
& h_{1}^{\prime}=\operatorname{atan} 2\left(b_{1}^{*}, a_{1}^{\prime}\right) \bmod 360^{\circ}, h_{2}^{\prime}=\operatorname{atan} 2\left(b_{2}^{*}, a_{2}^{\prime}\right) \bmod 360^{\circ} \\
& \Delta h^{\prime}= \begin{cases}h_{2}^{\prime}-h_{1}^{\prime}\left|h_{1}^{\prime}-h_{2}^{\prime}\right| \leq 180^{\circ} \\
h_{2}^{\prime}-h_{1}^{\prime}+360^{\circ}\left|h_{1}^{\prime}-h_{2}^{\prime}\right|>180^{\circ}, \quad h_{2}^{\prime} \leq h_{1}^{\prime} \\
h_{2}^{\prime}-h_{1}^{\prime}-360^{\circ}\left|h_{1}^{\prime}-h_{2}^{\prime}\right|>180^{\circ}, h_{2}^{\prime}>h_{1}^{\prime}\end{cases} \\
& \Delta H^{\prime}=2 \sqrt{C_{1}^{\prime} C_{2}^{\prime}} \sin \left(\Delta h^{\prime} / 2\right), \bar{H}^{\prime}=\left\{\begin{array}{cc}
\left(h_{1}^{\prime}+h_{2}^{\prime}+360^{\circ}\right) / 2 & \left|h_{1}^{\prime}-h_{2}^{\prime}\right|>180^{\circ} \\
\left(h_{1}^{\prime}+h_{2}^{\prime}\right) / 2 & \left|h_{1}^{\prime}-h_{2}^{\prime}\right| \leq 180^{\circ}
\end{array}\right.
\end{aligned}
$$

$$
\begin{aligned}
& T=1-0.17 \cos \left(\bar{H}^{\prime}-30^{\circ}\right)+0.24 \cos \left(2 \bar{H}^{\prime}\right) \\
& +0.32 \cos \left(3 \bar{H}^{\prime}+6^{\circ}\right)-0.20 \cos \left(4 \bar{H}^{\prime}-63^{\circ}\right)
\end{aligned}
$$


$S_{L}=1+\frac{0.015(\bar{L}-50)^{2}}{\sqrt{20+(\bar{L}-50)^{2}}}, S_{C}=1+0.045 \bar{C}^{\prime}, S_{H}=1+0.015 \bar{C}^{\prime} T$

$$
R_{T}=-2 \sqrt{\frac{\bar{C}^{\prime 7}}{\bar{C}^{\prime 7}+25^{7}}} \sin \left[60^{\circ} \cdot \exp \left(-\left[\frac{\bar{H}^{\prime}-275^{\circ}}{25^{\circ}}\right]^{2}\right)\right]
$$

$$
\Delta E_{00}=\sqrt{\left(\frac{\Delta L^{\prime}}{k_{L} S_{L}}\right)^{2}+\left(\frac{\Delta C^{\prime}}{k_{C} S_{C}}\right)^{2}+\left(\frac{\Delta H^{\prime}}{k_{H} S_{H}}\right)^{2}+R_{T} \frac{\Delta C^{\prime}}{k_{C} S_{C}} \frac{\Delta H^{\prime}}{k_{H} S_{H}}}
$$

Each of the $\Delta E$ values was calculated by a $\Delta E$ Calculator, which can also use RGB data (http://colormine.org/deltae-calculator/cie2000), and an average $\Delta E$ value was obtained for each abstract image. According to the calculations of 19 abstract images, the $\Delta E$ values varied between 4.50 and 79.53 (see Table 2). One of the abstract image's average $\Delta E$ value was 4.50 , which is perceptible at a glance, and the rest of the 19 abstract images' $\Delta E$ values were greater than 11 , which means the difference between colors used were perceptible or apparent (see Table 1). The $\Delta E$ values of 19 abstract images varied in order to understand the association between visual complexity and color as hypothesized before.

\section{3 | Preparing the questionnaire and procedures}

We prepared a questionnaire consisting of 38 questions to explore the effect of $\Delta E$ values of colors in an abstract image on visual complexity and visual interest. Participants were asked to rate the randomly ordered 19 generated abstract images according to the level of visual complexity and visual interest with a 5 -point scale $(1=$ least, $5=$ most $)$ on a color-calibrated computer screen (Intel Core i7 HD Graphics $2.2 \mathrm{GHz}$ ) in a windowless controlled lab environment. The experiment was conducted under controlled lighting conditions without daylight penetration.

T A B L E 1 Association between $\Delta E$ values and human perception

\begin{tabular}{ll}
$\boldsymbol{\Delta} \boldsymbol{E}$ values & Human perception \\
$\leq 1$ & Barely to Not perceptible \\
$1-2$ & Perceptible through close observation \\
\hline $2-10$ & Perceptible at a glance \\
\hline $11-49$ & Difference between color pairs are perceptible \\
\hline $50-100$ & Color pairs are in strong contrast \\
\hline
\end{tabular}

\section{4 | Sample group}

The sample group for the study consisted of 120 undergraduate students (first, second, third, and fourth year) from the Faculty of Art, Design and Architecture of Bilkent University, Ankara, Turkey. The age range of the students was from 18 to 28 years (mean age 21.3 years) and included both females (86 female) and males (34 male), all with full corrected vision. In addition, none of the students indicated they were color defective or color deficient. Each student has a color education within the scope of Faculty of Art, Design and Architecture courses.

\section{3 | RESULTS AND DISCUSSION}

It was hypothesized that, as the average $\Delta E$ value of color difference in an abstract image increases, visual interest and visual complexity will increase until reaching a threshold where visual interest and visual complexity start to decrease. In order to test the relationship between $\Delta E$ values of the color difference, visual interest, and visual complexity, 120 design students rated the 19 generated abstract images. According to the results of an analysis of variance test, the students' ratings for each of the 19 abstract images are significantly different from each other in terms of visual interest $(F[2261,18]=24.67, P=.000)$ and visual complexity $(F[2261,18]=54.15, P=.000)$. Thus, with the previously mentioned significant results, it was possible to analyze students' ratings statistically for each abstract image.

According to the results of students' ratings on a 5-point scale, the abstract image "5-6" was rated as the most visually complex and visually interesting one of the other abstract images, and "1-2" was rated as the least visually complex and visually interesting (see Table 2). As this particular study sought to find the relationship between $\Delta E$ values of the color difference used in abstract images and visual complexity and visual interest, the abstract images (1-2 and 1-3) with $\Delta E$ values of 4.50 and 11.25 were rated as the least visually complex and the least visually interesting, whereas the abstract images (5-6 and 6-5) with the $\Delta E$ value of 56.9 were rated as the most visually complex and the most visually interesting among other images. The abstract images (3-5 and 5-3) with the highest $\Delta E$ value of 79.5 had a moderate rating of visual interest and visual complexity (see Figure 3 ).

The abstract images rated as the least visually interesting and the complex ones, "1-2" and "1-3," had a monochromatic color scheme with tints of green that could be perceptible or distinguishable at first glance (see Table 1). Thus, having a single hue and its tints makes an abstract image less interesting and less complex. It is followed by the abstract images " $1-4$ " and "3-4," which had an analogous color scheme with green and yellow that allows them to be rated at a moderate level of 
T A B LE 2 The 19 generated abstract images using the colors from six different zones of CIE Chromaticity Chart and their average $\Delta E$, visual interest, and visual complexity values

\begin{tabular}{|c|c|c|c|c|}
\hline \multicolumn{5}{|l|}{ 1-2 (Monochromatic) } \\
\hline Average $\Delta E: 4.5$ & $1-3$ & $1-4$ & $1-5$ & $1-6$ \\
\hline V. Interest: 1.62 & (Monochromatic) & (Analogous) & (Analogous) & (Complementary) \\
\hline \multirow[t]{3}{*}{ V. Complexity: 1.99} & Average $\Delta E: 11.2$ & Average $\Delta E: 18.0$ & Average $\Delta E: 65.7$ & Average $\Delta E: 69.2$ \\
\hline & V. Interest: 2.19 & V. Interest: 2.46 & V. Interest: 2.84 & V. Interest: 2.90 \\
\hline & V. Complexity: 2.05 & V. Complexity: 2.20 & V. Complexity: 2.92 & V. Complexity: 2.90 \\
\hline Q & & & & \\
\hline \multirow{5}{*}{$\begin{array}{l}\mathbf{2 - 3} \\
\text { (Analogous) } \\
\text { Average } \Delta E: 63.3 \\
\text { V. Interest: } 2.96 \\
\text { V. Complexity: } 2.92\end{array}$} & $2-4$ & $2-5$ & $2-6$ & $3-4$ \\
\hline & (Analogous) & (Analogous) & (Split-complementary) & (Analogous) \\
\hline & Average $\Delta E: 69.7$ & Average $\Delta E: 61.7$ & Average $\Delta E: 61.1$ & Average $\Delta E: 24.6$ \\
\hline & V. Interest: 2.92 & V. Interest: 2.78 & V. Interest: 3.51 & V. Interest: 2.87 \\
\hline & V. Complexity: 3.23 & V. Complexity: 2.91 & V. Complexity: 3.68 & V. Complexity: 2.47 \\
\hline (i) & & & & \\
\hline \multirow{5}{*}{$\begin{array}{l}\text { 3-5 } \\
\text { (Triadic) } \\
\text { Average } \Delta E: 79.5 \\
\text { V. Interest: } 3.01 \\
\text { V. Complexity: } 3.57\end{array}$} & $3-6$ & $4-5$ & 4-6 & $5-3$ \\
\hline & (Complementary) & (Analogous) & (Complementary) & (Triadic) \\
\hline & Average $\Delta E: 71.7$ & Average $\Delta E: 77.8$ & Average $\Delta E: 71.3$ & Average $\Delta E: 79.5$ \\
\hline & V. Interest: 3.07 & V. Interest: 2.84 & V. Interest: 3.13 & V. Interest: 2.98 \\
\hline & V. Complexity: 3.32 & V. Complexity: 3.26 & V. Complexity: 3.48 & V. Complexity: 3.51 \\
\hline \multirow{2}{*}{$\begin{array}{l}0 . \\
5-6\end{array}$} & & & & \\
\hline & $6-2$ & $6-4$ & $6-5$ & \\
\hline (Tetradic) & (Split-complementary) & (Complementary) & (Tetradic) & \\
\hline Average $\Delta E: 56.9$ & Average $\Delta E: 61.1$ & Average $\Delta E: 71.3$ & Average $\Delta E: 56.9$ & \\
\hline V. Interest: $\underline{\mathbf{3 . 8 1}}$ & V. Interest: 3.22 & V. Interest: 3.15 & V. Interest: 3.76 & \\
\hline V. Complexity: $\underline{4.29}$ & V. Complexity: 3.51 & V. Complexity: 3.55 & V. Complexity: 4.13 & \\
\hline
\end{tabular}

The underlined values are showing the highest student ratings for visual complexity and visual interest.

visual interest and complexity. In those two abstract images, colors were more similar than the opposite but not as similar as a monochromatic scheme (see Table 1). Then, the other analogous color-schemed abstract images with blue and green, "1-5, 2-3, 2-4, 2-5, 4-5", were distinguished from the other analogous color-schemed images as they had higher average $\Delta E$ values ( $\geq 50$ ). The abstract images "1-6, 3-6, 4-6, 6-4" had a complementary color scheme with red and green. These images are followed by abstract images " $3-5,5-3$ " in terms of visual interest and visual complexity, which had a triadic color
F I GURE 3 The rating results of 19 generated abstract images by design students according to visual interest and visual complexity and its relationship between average $\Delta E$ values

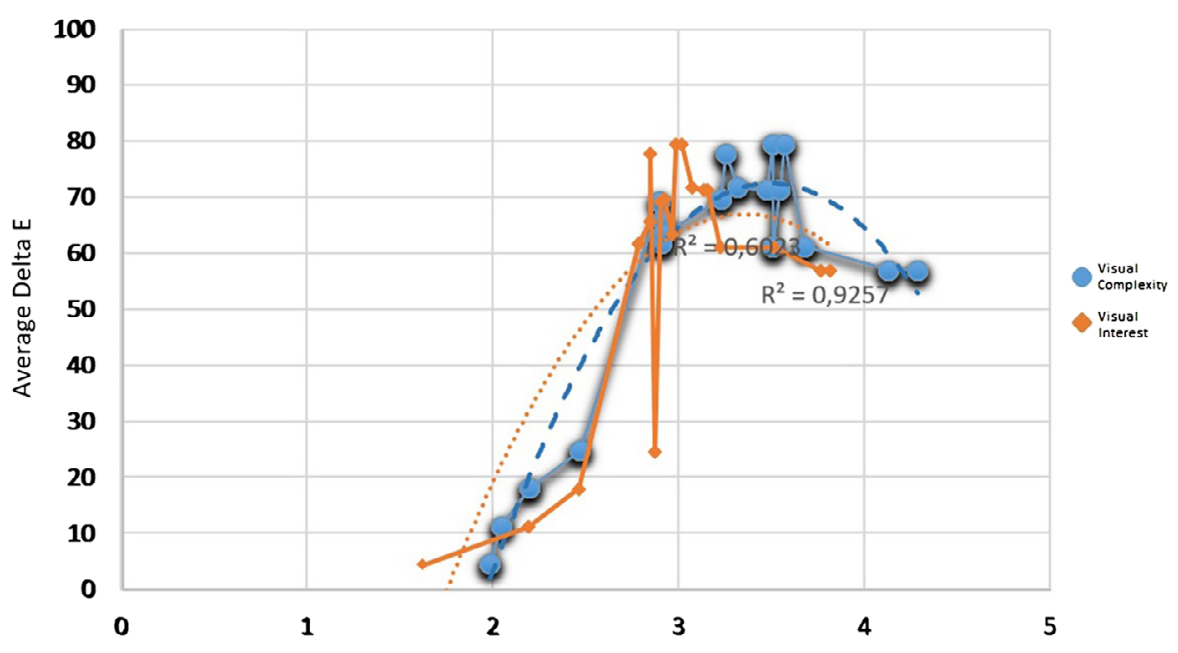


scheme with purple, purplish blue, and green. Afterward, the abstract images "2-6, 6-2" had a split-complementary color scheme with red and green, rated as the second most visual interesting and complex images. The most visually interesting and visually complex abstract images were "5-6, 6-5," with a tetradic color scheme with blue, purplish blue, yellow, and yellowish orange.

As Figure 3 shows, there is a positive but weak correlation between average $\Delta E$ values of visual interest (Pearson correlation $r[19]=0.631, P=.004)$ and average $\Delta E$ values of visual complexity (Pearson correlation $r[19]=0.735, P=.000$ ). A graphical analysis demonstrates an inverted U-curve relationship between average $\Delta E$ values and visual complexity, which was similar to the relationship between average $\Delta E$ values and visual interest (see Figure 3). Berlyne ${ }^{7}$ showed that complexity was a dominant determinant of interestingness and pleasingness of a stimulus ${ }^{7-9}$ and suggested that the relationship between complexity and pleasingness could be explained by an inverted U-curve. This concept of an optimal amount of stimulus complexity has been supported by numerous studies that found an inverted U-curve when characterizing aesthetic preference as a function of complexity. ${ }^{9-14}$ Most of the prior visual complexity and visual interest studies did not consider the role of color both on complexity and interest, and the ones that considered color used isolated color patches and did not measure it as a whole. In addition, individual differences such as age and education level were considered problems in previous complexity studies, and in this study, those problems were eliminated as design students of equivalent age were selected as participants, and they all had the same understanding of design education of artistic tendencies and creativity. Thus, this study demonstrated the relationship between color, visual interest, and visual complexity by adding color and representing it with a quantitative value $(\Delta E)$ in the complexity field; this created a new understanding that was pioneered by Berlyne. ${ }^{9}$

To conclude, with the results of this study, a quantitative and objective knowledge about the association between color and visual complexity was obtained. This study can be used as a guide by those who want to generate visually complex and interesting images using colors.

\section{ACKNOWLEDGMENTS}

This research was conducted as a part of a $\mathrm{PhD}$ thesis of the first author, Rengin Kocaoğlu Aslanoğlu, at Bilkent Üniversitesi.

\section{ORCID}

Rengin Kocaoğlu Aslanoğlu (D) https://orcid.org/0000-00022963-4299

Nilgün Olguntürk (1) https://orcid.org/0000-0001-7947-1101

\section{REFERENCES}

[1] Ou LC, Luo MR, Woodcock A, Wright A. A study of colour emotion and colour preference. Part II: colour emotions for twocolour combinations. Color Res Appl. 2004;29(4):292-298.

[2] Kocaoğlu R, Olguntürk N. Color and visual complexity in abstract images. Color Res Appl. 2018;43:1-6.

[3] Nadal M, Munar E, Marty G, Cela-conde CJ. Visual complexity and beauty appreciation: explaining the divergence of results. Empir Stud Arts. 2010;28:173-191.

[4] Backhaus W, Kliegl R, Werner JS. Color Vision: Perspectives from Different Disciplines. Walter de Gruyter. Berlin, Germany; 1998:188.

[5] Sharma G, Wu W, Dalal EN. The CIEDE2000 color-difference formula: implementation notes, supplementary test data, and mathematical observations. Color Res Appl. 2005;30(1):21-30.

[6] ISO/CIE 11664-6:2014(E) Colorimetry-Part 6: CIEDE2000 Colour-Difference Formula. Vienna, Austria: CIE; 2014.

[7] Berlyne DE. Complexity and incongruity variables as determinants of exploratory choice and evaluative ratings. Can J Psychol. 1963;17:274-290.

[8] Berlyne DE, Ogilvie JC, Parham LC. The dimensionality of visual complexity, interestingness, and pleasingness. Can J Psychol. 1968;22:376-387.

[9] Berlyne DE. Aesthetics and Psychobiology. NewYork, NY: Appleton- Century-Crofts; 1971.

[10] Vitz PC. Preference for different amounts of visual complexity. Behav Sci. 1966;11:105-114.

[11] Saklofske DH. Aesthetic complexity and exploratory behavior. Percept Mot Skills. 1975;41:363-368.

[12] Farley FH, Weinstock CA. Experimental aesthetics: children's complexity preference in original art and photo reproductions. Bull Psychon Soc. 1980;15:194-196.

[13] Imamoglu Ç. Complexity, liking and familiarity: architecture and non- architecture Turkish students' assessments of traditional and modern house facades. J Environ Psychol. 2000;20:5-16.

[14] Güçlütürk Y, Jacobs RHAH, van Lier R. Liking versus complexity: decomposing the inverted U-curve. Front Hum Neurosci. 2016;10:112.

\section{AUTHOR BIOGRAPHIES}

Rengin Kocaoğlu Aslanoğlu works as a researcher ( $\mathrm{PhD}$ Candidate) in the Interior Architecture and Environmental Design Department at Bilkent University Ankara, Turkey. Her research interests include perception of color and effects of artificial lighting on attention and performance.

Nilgün Olguntürk is an associate professor at the Department of Interior Architecture and Environmental Design, the Faculty of Art, Design and Architecture, Bilkent University, Ankara. She received BArch, MA, and $\mathrm{PhD}$ degrees in architecture. Her professional experience has included appointments as instructor at the Middle East Technical University and research fellow at the London South Bank University. She has worked on research projects in the United Kingdom for NHS Estates 
(Department of Health) on color design in hospitals and EPSRC/DTLR LINK (Department of Transport, Local Government and the Regions) on color, visual impairment, and transport environments. She has 20 years of research experience on color perception, color preference, and color use in architecture. Her current research and teaching include color and lighting and undergraduate and graduate studios. Her work has appeared in various journals such as Color Research and Application, Optics and Laser Technology, Building and Environment, Architectural Science Review, and Journal of Imaging Science and Technology. Dr. Olguntürk is, among others, an active member of the International
Commission of Illumination (CIE); the International Colour Association (AIC); the Inter-Society Color Council (ISCC); and the Uluslararası Mimarlar Birliği International Union of Architects (UIA), Chamber of Architects of Turkey.

How to cite this article: Kocaoğlu Aslanoğlu R, Olguntürk N. Color and visual complexity in abstract images: Part II. Color Res Appl. 2019;44:941-947. https://doi.org/10.1002/col.22408 